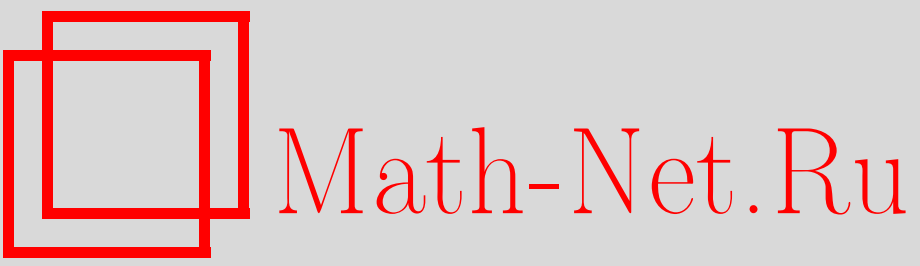

В. Н. Кривцов, Логики творящего субъекта: консервативность относительно интуиционистского исчисления предикатов, УМН, 1996, том 51, выпуск 1, 151-152

DOI: https://doi.org/10.4213/rm928

Использование Общероссийского математического портала Math-Net.Ru подразумевает, что вы прочитали и согласны с пользовательским соглашением

http://www.mathnet.ru/rus/agreement

Параметры загрузки:

IP: 54.198 .187 .58

26 апреля 2023 г., 05:15:59 


\title{
ЛОГИКИ ТВОРЯЩЕГО СУБЪЕКТА: \\ КОНСЕРВАТИВНОСТЬ ОТНОСИТЕЛЬНО ИНТУИЦИОНИСТСКОГО ИСЧИСЛЕНИЯ ПРЕДИКАТОВ
}

\author{
В.Н. Кривцов
}

В работе [1] была определена семантика для языка $L$, получающегося в результате присоединения к языку исчисления предикатов Гейтинга (HPC) логического оператора $\square$ и расширения стандартного определения понятия формулы путем добавления пункта: если $A$ - формула и $b$ - параметр, то $\square_{b} A$ - формула. Там же была установлена семантическая полнота исчислений CS-W и CS-S, содержаших основные аксиомы теории творящего субъекта.

В настоящей работе рассматриваются расширения указанных исчислений путем добавления следующих схем аксиом (подробное обсуждение расширений аксиоматики Крайзеля можно найти в [2] и [3]):

(1) $\forall x\left(\square_{x}(A \vee B) \rightarrow\left(\square_{x} A \vee \square_{x} B\right)\right)$,

(2) $\forall x\left(\left(\square_{x} A \vee \square_{x} B\right) \rightarrow \square_{x}(A \vee B)\right)$,

(3) $\forall x\left(\square_{x}(A \& B) \leftrightarrow\left(\square_{x} A \& \square_{x} B\right)\right)$,

(4) $\forall x\left(\square_{x}(A \rightarrow B) \rightarrow\left(\square_{x} A \rightarrow \square_{x} B\right)\right)$,

(5) $\forall x\left(\square_{x} B \rightarrow \square_{x}(A \rightarrow B)\right)$,

(6) $\forall x\left(\square_{x} \exists y A(y) \leftrightarrow \exists y \square_{x} A(y)\right)$,

(7) $\forall x \forall y\left(\square_{x} \square_{y} A \rightarrow \square_{x} A\right)$,

(8) $\forall x \forall y\left(\left(\square_{x} A \& \square_{y} A\right) \rightarrow\left(\square_{x} \square_{y} A \vee \square_{y} \square_{x} A\right)\right)$,

(9) $\forall x\left(\forall y \square_{x} A(y) \rightarrow \square_{x} \forall y A(y)\right)$,

(10) $\forall x\left(\square_{x} \forall y A(y) \rightarrow \forall y \square_{x} A(y)\right)$.

Пусть PEM обозначает схему аксиом $A \vee \neg A$, a DNS ("double negation shift") - cхему $\forall x \neg \neg A(x) \rightarrow \neg \neg \forall x A(x)$.

Справедливы следующие утверждения (в формулировке теоремы 1 PEM и DNS - схемы в языке $L)$.

Teopema 1. (1) CS-S + (1) + (10) |-PEM.

(2) $\mathbf{C S}-\mathbf{W}+(1)+(10) \mid-\mathbf{D N S}$.

Tеорема 2. (1) CS-S $+(1)+\cdots+(9)-$ консервативное расширение $\mathbf{H P C}$.

(2) CS-W $+(1)+\cdots+(10)$ - консервативное расширение $\mathbf{H P C}+$ DNS.

Tеорема 3. CS-S $+(2)+\cdots+(10)-$ консервативное расширение HPC.

Теорема 4. Пусть $\mathbf{C S}_{\mathbf{3}}^{\mathbf{S}}(-\forall)$ обозначает схему $\mathbf{C S}_{\mathbf{3}}^{\mathbf{S}}$ для формул, не содержсащих $\forall$. Тогда:

(1) $\mathbf{H P C}+\mathbf{C S}_{\mathbf{1}}+\mathbf{C S}_{\mathbf{2}}+\mathbf{C S}_{\mathbf{3}}^{\mathbf{S}}(-\forall)+(1)+\cdots+(10)-$ консервативное расширение HPC.

(2) $\mathbf{C S}-\mathbf{W}+\mathbf{C S}_{\mathbf{3}}^{\mathbf{S}}(-\forall)+(1)+\cdots+(10)-$ консервативное расширение $\mathbf{H P C}+\mathbf{D N S}$

Теорема 5. Пусть $\mathbf{C S}_{\mathbf{3}}^{\mathbf{S}}(-\vee)$ обозначает схему $\mathbf{C S}_{\mathbf{3}}^{\mathbf{S}}$ для формул, не содержащих $\vee$. Tогда:

(1) $\mathbf{H P C}^{\prime}+\mathbf{C S}_{\mathbf{1}}+\mathbf{C S}_{\mathbf{2}}+\mathbf{C S}_{\mathbf{3}}^{\mathbf{S}}(-\mathrm{V})+(1)+\cdots+(10)-$ консервативное расширение HPC.

(2) $\mathbf{C S}-\mathbf{W}+\mathbf{C S}_{\mathbf{3}}^{\mathbf{S}}(-\mathrm{V})+(1)+\cdots+(10)-$ консервативное расширение $\mathbf{H P C}+\mathbf{D N S}$.

Теоремы $2-5$ интересны, главным образом, с точки зрения анализа основного отношения $\square_{b} A$; самостоятельный интерес представляют теоретико-модельные конструкции, используемые в доказательствах этих теорем, поскольку они допускают расширение до моделей аналитического языка с оператором $\square$, и, следовательно, до моделей систем интуиционистского анализа в которых истинна схема Крипке.

В качестве примера приведем набросок доказательства теоремы 2. (Используемые далее обозначения совпадают с теми, которые применялись в работе [1].) 
Для доказательства теоремы 2 (1) достаточно показать, что каждую модель Бета $\mathfrak{B}=\langle T, \mathbb{N}, \varphi\rangle$ теории $\mathbf{H P C}$ можно расширить до $\mathbf{C S - S - м о д е л и ~} \mathfrak{B}^{*}=\langle T, \mathbb{N}, f, \varphi\rangle$, такой, что в $\mathfrak{B}^{*}$ истинны схемы $(1), \ldots,(9)$, следовательно, достаточно подходящим образом задать функцию $f: T \times$ $S t_{\mathbb{N}} \times \mathbb{N} \rightarrow\{0,1,2\}$.

Приведем индуктивное определение $f$ :

(i) Если $A$ атомарная или имеет вид $\forall x B$, то:

$$
f(k, A, n)=\left\{\begin{array}{l}
2, \text { если } \operatorname{lth}(k) \geqslant n \text { и } k^{*}(n) \Vdash A ; \\
0, \text { если } \operatorname{lth}(k)<n ; \\
1 \text { в остальных случаях; }
\end{array}\right.
$$

(ii) $f\left(k, A_{1} \& A_{2}, n\right)=\min \left\{f\left(k, A_{i}, n\right): i \in\{1,2\}\right\}$;

(iii) $f\left(k, A_{1} \vee A_{2}, n\right)=\max \left\{f\left(k, A_{i}, n\right): i \in\{1,2\}\right\}$;

(iv) $f\left(k, A_{1} \rightarrow A_{2}, n\right)= \begin{cases}2, & \text { если } \operatorname{lth}(k) \geqslant n, \quad k^{*}(n) \Vdash A_{1} \rightarrow A_{2} \text { и } \\ & \left(k^{*}(n) \Vdash \square_{n} A_{1} \Rightarrow k^{*} \Vdash \square_{n} A_{2}\right) ; \\ 0, & \text { если } \operatorname{lth}(k)<n ; \\ 1 \quad \text { в остальных случаях; }\end{cases}$

(v) $f\left(k, \square_{m} A_{1}, n\right)=\left\{\begin{aligned} 2, & \text { если } \operatorname{lth}(k) \geqslant n, \quad f(k, A, n)=2 \text { и } \\ & k^{*}(n) \Vdash \square_{m} A_{1} ; \\ 0, & \text { если } \operatorname{lth}(k)<n ; \\ 1 & \text { в остальных случаях; }\end{aligned}\right.$

(vi) $f\left(k, \exists x A_{1}(x), n\right)=\max \left\{f\left(k, A_{1}(m), n\right): m \in \mathbb{N}\right\}$.

Здесь $k^{*}(n)$ обозначает кортеж $k^{\prime} \leqslant k$ такой, что $\operatorname{lth}\left(k^{\prime}\right)=n$ (для $k$ таких, что $\left.\operatorname{lth}(k) \geqslant n\right)$.

Аналогичная конструкция используется в доказательстве теоремы $2(2)$ : модели Бета $\mathfrak{B}=$ $\langle T, \mathbb{N}, \varphi\rangle$ теории $\mathbf{H P C}+\mathbf{D N S}$ расширяются до CS-W-моделей $\mathfrak{B}^{*}=\left\langle T, \mathbb{N}, f_{1}, \varphi\right\rangle$, где $f_{1}$ определяется так же, как $f$, за исключением того, что теперь мы полагаем:

$$
f_{1}\left(k, \forall x A_{1}(x), n\right)=\min \left\{f_{1}\left(k, A_{1}(m), n\right): m \in \mathbb{N}\right\} .
$$

В заключение отметим, что открытой является следующая проблема: в какой мере результаты о консервативности для НРС, сформулированные в теоремах $2-5$, переносятся на случай арифметики Гейтинга НА?

\section{СПИСОК ЛИТЕРАТУРЫ}

[1] Кривцов В.Н.// УМН. 1994. №6. С. 205-206. [2] van Rootselaar B. Intuitionism and Proof Theory. Amsterdam: North-Holland, 1970. [3] Dummett M. A. E. Elements of Intuitionism. Oxford: Clarendon Press, 1977. 